\title{
Validez de instrumento para medir el aprendizaje creativo $^{1}$
}

\author{
Validity of Instrument to measure Creative Learning \\ Mawency Vergel Ortega $\quad$ José Joaquín Martínez \\ mawency@ufps.edu.co_josemartinez@ufps.edu.co \\ José Francisco Nieto ${ }^{c}$ \\ josenieto@ufps.edu.co
}

\begin{abstract}
Resumen
El artículo presenta la validez y fiabilidad del instrumento CREA, cuyo objetivo es medir el aprendizaje creativo de estudiantes que cursan programas académicos de pregrado en el municipio de Cúcuta, Colombia. Sigue un diseño de estudio descriptivo de validación de escalas multidimensionales a través de análisis factorial, validez de constructo, y facial. La población estudio la constituyen 23564 estudiantes de programas de pregrado ofertados por universidades del municipio de San José de Cúcuta, constituida la muestra por 595 estudiantes con edades comprendidas entre 18 y 25 años. Se obtuvo una escala de 35 ítems. El análisis factorial arrojó dimensiones de práctica pedagógica orientada al liderazgo, pensamiento creativo, satisfacción emocional, independencia, actitud creativa, productividad, competencias en liderazgo, motivación; la consistencia interna fue adecuada (alfa de Cronbach 0,72-0,95), coeficiente de correlación intraclase global de 0,967 (IC del $95 \%, 0,933-0,984$ ), promedio de diferencias en sumatoria de ítems -1,3 (DS: $8,5)$ e índices de Kappa superiores a 0,9, lo cual muestran un alto acuerdo entre expertos. Se concluye que CREA es un instrumento válido y confiable para el contexto Cúcuta, Colombia.
\end{abstract}

Palabras clave: creatividad, capacidades, validez de pruebas, juventud, fiabilidad.

\section{Abstract}

${ }^{1}$ Vergal, M., Martínez J., Nieto, J. (2016) Validez de instrumento para medir el aprendizaje creativo. Comunicaciones en Estadística, 9(2), 239-254.

${ }^{a}$ Universidad Francisco de Paula Santander, Colombia.

${ }^{\mathrm{b}}$ Universidad Francisco de Paula Santander, Colombia.

${ }^{\mathrm{c}}$ Universidad Francisco de Paula Santander, Colombia. 
The article presents the validity and reliability of CREA instrument, its objective is to evaluate the creative learning students pursuing undergraduate academic programs in the city of Cúcuta, Colombia. Follows a descriptive study design validation through multidimensional scaling factor analysis, construct validity, and facial. The study population were students of undergraduate engineering, management and agricultural sciences aged between 18 and 25 years in the city of Cucuta, Colombia, the sample constituted by 595 students. A scale of 35 items was obtained. Factor analysis showed dimensions of teaching practice-oriented leadership, creative thinking, emotional fulfillment, independence, creative attitude, productivity, leadership skills, motivation; Internal consistency was adequate (Cronbach's alpha 0.72 to 0.95 ), intraclass correlation coefficient of 0.967 overall (95\% CI, 09330984), differences in average sum of items -1.3 (SD 8.5) and Kappa indices greater than 0.9, it shows high agreement among experts. It is concluded that CREA is a valid and reliable for the context Cucuta, Colombia instrument.

Keywords: Creativity, capabilities, test validity, youth, reliability.

\section{Introducción}

La relación entre los constructos creatividad y aprendizaje es un tópico que, a pesar de ser estudiado, requiere de claridad, teniendo en cuenta el contexto y los continuos cambios en ciencia y tecnología que demanda la sociedad actual. Tradicionalmente se había asumido que personas creativas eran personas inteligentes y, por consiguiente, eran personas que lograban el desarrollo de competencias o un alto rendimiento académico en el proceso de aprendizaje de diferentes ciencias. Otros planteamientos establecieron que la existencia de diferentes grados de aptitud cognitiva establecía diferentes niveles de creatividad, pero independientes de la inteligencia (Wallack \& Koga 1965). Torrance (1976), partiendo del criterio de que la inteligencia y la creatividad eran constructos diferentes, postula que cuando el cociente intelectual está por debajo de cierto límite, la creatividad también se encuentra limitada, mientras que, cuando el cociente intelectual se posiciona por encima de cierto límite $(C I>115)$ la creatividad llega a ser una dimensión independiente del cociente intelectual.

Por otra parte, hipótesis en psicología y en el ámbito educativo mundial dieron lugar a la teoría de las inteligencias múltiples, en la cual ciertos planteamientos teóricos de Gardner (1983) postularon que constructos creatividad e inteligencia son lo mismo; propuso Gardner (1999) un razonamiento en torno al aprendizaje apoyado en la creatividad como perspectiva interactiva desde las inteligencias múltiples y en niveles que tienen en cuenta la persona, la disciplina y el ámbito circundante emitiendo juicios sobre la validez y calidad tanto del individuo como de sus productos (Bermejo et al. 2010); planteamientos donde el constructo creatividad, aprendizaje y aprendizaje creativo, en el campo de las interacciones humanas, ha evolucionado desde la complejidad como elemento emergente constituyéndose en constructos transversales al ser humano y a la sociedad del cual Cabrera \& 
de la Herrán (2015) manifiestan debe continuar investigándose en formación y autoformación para la creatividad de estudiantes, docentes e investigadores con el propósito de ampliar ámbitos de conciencia colectiva y dar respuesta tanto a indicadores en educación e innovación como a la solución de problemas de la sociedad y a la explosión de conocimiento científico desde procesos de formación de estudiantes.

Esta explosión de conocimiento científico que progresa por eliminación del error requiere cambios en sus estructuras mentales, de una formación en y para la generación de conocimiento científico; requiere también de cambios en los currículos de las instituciones educativas, donde la ciencia se adhiera a la pedagogía, donde saber intelectual y capacidad social se constituyen en un fin educativo, donde instituciones educativas se den a la tarea de fomentar la creatividad, de enseñar el conocimiento a través de una respuesta reflexiva que reoriente sus prácticas vinculando al estudiante no solo como ser pasivo, sino como un ser con potencialidades, competencias, habilidades, que debe explotar y asociar a lo enseñado; debe para ello conocerle, caracterizarlo y evaluar todas sus potencialidades.

Según el índice global de creatividad 2015 del Martin Prosperity Institute (Dutta et al. 2015), Colombia ocupa el puesto 71, por debajo de Venezuela, que se encuentra en el 61, y muy rezagada de Ecuador que se ubica en el ranking de 44, entre 139 naciones del planeta; en tecnología mereció el puesto 89, pero en talento se ubicó en el puesto 75, en tolerancia y en innovación mejoró, teniendo en cuenta el índice global de innovación; no obtiene, tiene grandes desafíos en materia de innovación, si desea disminuir la brecha que persiste con las economías del primer mundo.

Lo anterior refleja la necesidad de integrar miradas objetivas y subjetivas, que permitan comprender y evaluar la acción creadora del estudiante, pues esto podría ayudar a entes gubernamentales, a priorizar políticas educativas en innovación desde el aprendizaje creativo, revelando la necesidad de combinar las dimensiones que forman parte de este. Medir a estudiantes, profesores y a las universidades es importante, tal como lo manifiesta Parody (2015), pues da información a padres y jóvenes en la toma de decisiones.

Por otra parte, la creatividad como factor de calidad de programas e instituciones es un elemento importante de evaluar, en términos de dimensiones acorde con modelos como el modelo multidimensional de calidad MIDE, el cual, aunque cuestionado, vincula indicadores de creatividad en desempeño (competencias específicas, lectura crítica), docencia, investigación (investigadores, obras, artículos, patentes), internacionalización (coautorías), presencia y atracción (Observatorio de la Universidad Colombiana 2015), así como un elemento para identificar percepciones y valor que la juventud da a la creatividad teniendo en cuenta el contexto en el cual estudia.

Este problema puede observarse por componentes elementales evidenciando subutilización de espacios disponibles para la creación, así como la carencia de una red articulada y eficiente de espacios el desarrollo de la ciencia desde el despertar de la creatividad en jóvenes en el aula, esparcimiento pasivo de semilleros, jóvenes ta- 
lentos no inmersos en procesos de innovación, prácticas pedagógicas tradicionales, no enmarcadas en un desarrollo real de competencias o de enfoques dialógico-críticos, como parte de un sistema integrado educativo, de ciencia y tecnología que dé respuesta a planteamientos inmersos en políticas y planes de desarrollo regionales y nacionales y de otros factores que se desconocen estén asociados al fenómeno y que se asocian a la innovación, para que, como lo manifiesta Wasserman (2015), el Ministerio de Educación, acompañe, abrace y estimule buenas iniciativas institucionales.

De esta manera, el problema posee consideraciones de orden social, educativo, de innovación, generación de conocimiento e internacionalización, demandando una formulación integral que dé respuesta a necesidades en torno al desarrollo de la creatividad en los jóvenes. En ese sentido, Bermejo et al. (2010) sostuvieron que no existen muchos instrumentos validados que midan el potencial creativo de estudiantes, lo cual hace que la identificación de jóvenes sobresalientes sea un problema. Para ello se diseña un instrumento que conlleve analizar el aprendizaje creativo en la juventud en la ciudad de Cúcuta.

Validar el instrumento CREA dará respuesta a esta problemática, puesto que las escalas genéricas permiten valorar actitudes de grupos en general; en cambio, las escalas específicas permiten analizar un fenómeno, problema u otras variables relacionadas con la creatividad del joven en el y durante el proceso de aprendizaje. La utilización de diferentes escalas y cuestionarios es útil para establecer estados, aplicar políticas sociales, así como la asignación de recursos según Vivant (2013).

\section{Metodología}

La investigación sigue un estudio descriptivo de validación de un instrumento. La población estudio la constituyen 23.564 estudiantes de programas académicos ofertados por universidades en el municipio de San José de Cúcuta, Colombia. Seleccionada la muestra a través de muestreo por conglomerados, la muestra la conforman 595 jóvenes entre 18 y 25 años, estudiantes de cálculo diferencial, ecuaciones diferenciales y física mecánica de las universidades Francisco de Paula Santander, Universidad de Santander, Fundación de Estudios Superiores Comfanorte (FESC), Instituciones tecnológicas de Santander, Escuela Superior de Administración Pública.

La investigación busca medir el constructo aprendizaje creativo de estudiantes que cursan programas académicos de pregrado en el municipio de Cúcuta, Colombia. Definido aprendizaje creativo desde planteamientos de Csikszentmihalyi (1996) como el conocimiento construido con la implicación activa del sujeto, desde su planificación hasta su internalización, caracterizado por la motivación intrínseca, estar centrado en el discente, carácter abierto del proceso de enseñanza, percibido como proceso que permite la asimilación, adquisición, transformación de la vida psíquica del comportamiento de la personalidad en los niveles de regulación inductora y ejecutora conducente a la solución creadora novedosa de diferentes 
problemas de la vida (Sáenz \& Larraz 2015).

Se diseñó un cuestionario de carácter multidimensional (Oyekale et al. 2009, Vergel et al. 2015), en el cual se evalúa si la riqueza de aglomeración se distribuye en forma justa, según participación en actividades de investigación, y si este factor impacta positivamente en su creatividad. Sigue un modelo de ecuaciones estructurales (?) y modelo de elección discreta siguiendo metodología de análisis desagregado por dimensiones e indicadores incluyendo el índice de creatividad global (Florida et al. 2009); medición basada en el talento, la tecnología y la tolerancia, la cual permite identificar variables que determinan la incidencia de medir el porcentaje de personas en la fuerza laboral de clase creativa, la capacidad de innovación, teniendo en cuenta las patentes registradas en un lugar específico, la industria de alta tecnología y la diversidad; así como variables de desarrollo de talento y ecosistema de aprendizaje, apertura, confianza. El modelo desagregado probabilístico considera el comportamiento individual, en lugar de zonas geográficas como unidad de observación, seleccionando de alternativas posibles aquella que maximiza su utilidad partiendo de la idea de que el aprendizaje creativo se genera en el proceso de toma de decisiones individual. Siguiendo el trabajo de Becker se cuentan con restricciones económicas y temporales, eligiendo tipos de inteligencias de los jóvenes $G$ y tiempo de ocio L que maximiza su utilidad, teniendo en cuenta si dedican mas horas de trabajo $t_{w}$ para incrementar su rendimiento académico $I$, según nivel de creatividad $w$, inversión en el aprendizaje $c_{j}$, tiempo para resolver un problema y adquirir más aprendizaje, formalmente se estableció:

$$
\text { máx } U(G, L) \text { sujeto a } G=I+w t_{w}-c_{j}, \mathrm{y} L=T-t_{w}-t_{j}
$$

El procedimiento seguido para la construcción de la escala fue el propuesto por Morales et al. (2003), siguiendo pasos como: definir constructo que se desea medir, redactar los ítems candidatos, teniendo en cuenta dimensiones de test de inteligencia, aprendizaje y creatividad, determinar el modo de respuesta y el número de respuestas adicionales, preparar la clave de corrección, preparar preguntas o instrumentos adicionales en previsión de los estudios para evaluar la validez de contenido (consulta a expertos, entrevista cognitiva, grupo focal), obtener lista refinada de ítems, analizar si discriminan y si se pueden considerar indicadores del mismo rasgo, obtener datos de una muestra, comprobar la fiabilidad general de la escala, seleccionar los ítems definitivos, comprobar la validez; el procedimiento incluyó la técnica del análisis factorial exploratorio y análisis factorial confirmatorio con la finalidad de lograr una reducción de la dimensionalidad de los datos analizados y obtener un conjunto de factores que facilitó la interpretación teórica de los hallazgos. El análisis factorial exploratorio implicó los siguientes pasos, de acuerdo con Pardo \& Ruiz (2002): verificación de supuestos, análisis de la matriz de correlaciones, análisis de la varianza explicada, obtención de la solución factorial y, finalmente, interpretación de los factores. Análisis factorial confirmatorio sigue el procedimiento lógica de SEM analizando la estructura de covarianza que compara la matriz de covarianza derivada de las variables observadas y la matriz de covarianzas reproducidas por el modelo. 
De igual manera, se incorporaron en la batería inicial variables de emprendimiento, exploración e innovación, usadas en el estudio Creative City Index (Landry \& Hyams 2012), actitudes del individuo creativo, manifiestas por Sternberg (2006), actividades como creencias, técnicas, orientación final al producto, control ambiental y uso de los sentidos de escala de Kumar et al. (1997) y componentes desde el modelo de Ambiele como destrezas adecuado, estilo cognitivo, conocimiento explícito e implícito para generar ideas novedosas, estilo de trabajo favorecedor (Garaigordobil \& Pérez 2002). Relacionado con el concepto de la generación de ideas el pensamiento creativo ya sea pensamiento divergente, brainstorming, pensamiento sinéctico o analógico, pensamiento asociativo, pensamiento de bisociación, pensamiento lateral, pensamiento janosiano (Ang \& Low 2000) y asociado a relaciones públicas según modelo de Green (2010) la información, incubación, integración e ilustración.

El instrumento vinculó un factor principal asociado a satisfacción con la productividad (5 ítems), un factor asociado a independencia (5 ítems); un tercer factor asociado a motivación (5 ítems). La variable flexibilidad, indicador clásico en la práctica pedagógica (Campbell et al. 1976), se incorpora dentro de factor pensamiento creativo (8 ítems). Asimismo, se utilizan variables que corresponden con dimensiones de actitud creativa ( 2 ítems), medida en una escala de 5 puntos en escala Likert, según el estudiante considere que se encuentra muy inferior al promedio del grupo, inferior, ocupa un lugar promedio, si se considera superior al promedio o muy superior al promedio del grupo en el cual estudia; incluye la evaluación cognitiva de dimensiones que cada persona considera más importante para su vida (Brown et al. 2004), medida en una escala de 5 puntos, desde 1 (completamente insatisfecho) a 5 (completamente satisfecho).

La productividad, medida a través de pregunta sobre dedicación a problemas, problemáticas, actividades que se le proponen en el aula, capacidad de dedicarse a la solución de problemas durante un largo intervalo de tiempo, no presentar desaliento cuando fracasa ante el desarrollo de una problemática, buscar otras alternativas que conlleven cumplir con actividades asignadas.

La independencia medida desde el establecimiento de prioridades para cumplir una tarea, emprender soluciones de manera individual y confrontar soluciones con equipo de trabajo en el aula, buscar nuevas vías para resolver un problema o desarrollar prototipos o simuladores, seleccionar formas novedosas y rechazar indicaciones de cómo debe resolver una problemática o tarea, evidenciar el esfuerzo ante los impedimentos que entorpecen la correcta solución de una tarea o problema.

La motivación, desde la participación con iniciativa y saber buscar información para la solución de problemas, el establecer nexos con compañeros para identificar fuentes de información, evidenciar interés por conocer lo que hacen otros grupos de estudiantes en otros lugares para ampliar su espectro de información sobre un tema científico técnico.

En subcategoría pensamiento creativo se aplican indicadores de forma (Torrance 1976, Cramond et al. 2005) en componente verbal y figurativo en que los jóvenes enuncian consecuencias a situación improbable, usos, formulación de cuestiones 
sobre una acción en imágenes donde se presenta una situación ambigua aplicada a la formación que están recibiendo (clase cálculo diferencial, física mecánica, ecuaciones diferenciales), se analizó acorde con el planteamiento de hipótesis, soluciones y métodos de solución propuestos; figurativo a partir de figuras curvas, funciones familias de funciones, los sujetos debían diseñar dibujos, composiciones de funciones, mosaicos a partir de traslaciones o rotaciones, modelar situaciones planteadas en el aula a través de gráficas; plantear soluciones a través del diseño de prototipos o modelos que permitan simular la solución. La evaluación de la producción consideró criterio de fluidez, flexibilidad, originalidad, enriquecimiento de ideas.

La flexibilidad inmersa en pensamiento creativo, medida desde el establecer diferentes vías de solución de un problema, no asumiendo como único el que el profesor estima, y que lo conduzca de manera exitosa al resultado final de este; estimar que los profesores deben ser abiertos ante diversas soluciones que los estudiantes propongan en la solución de un problema, sin que no se corresponda con lo planificado por el profesor con anterioridad; establecer nuevas actuaciones como jóvenes, diferentes al modelo de sus padres y profesores, pero estando dentro de las normas establecidas; seleccionar diferentes vías para estudiar, en correspondencia con las condiciones y la exigencia de actividades, obteniendo resultados satisfactorios y seleccionar varias vías de solución a los problemas que se enfrentan en la vida cotidiana.

Indicadores de actitud creativa relacionan el identificar otras opiniones, para cambiar sus puntos de vista ante criterios disonantes o contrarios a partir de las condiciones para la solución de un problema, emprender acciones que le permiten interactuar con sus compañeros ante valoraciones y operaciones relacionadas con la solución de una tarea o problema de tipo experimental; confusión ante el desorden; entender lo existente frente al desorden aunque le cuesta trabajo llegar a conclusiones claras; trabajo bien en el desorden, rápidamente saco conclusiones claras y entendiendo lo existente.

Se utilizaron variables de control: género, edad, estrato socioeconómico, nivel educativo (7 niveles) (González 2008), satisfacción con su formación teniendo en cuenta desarrollo de procesos de creación (1-5). La variable satisfacción con recursos emocionales, a través de ítems relacionados con rol, autoestima, reconocimiento, autonomía y afrontamiento (Prieto et al. 2008, Martínez et al. 2015), buscó medir la satisfacción por su capacidad para tomar decisiones, afrontarlas y controlar consecuencias, libertad para expresar opiniones, trato que recibe, su posición y reconocimiento en la sociedad y satisfacción consigo mismo.

El índice de bondad de ajuste del modelo se evaluó a través del test de HosmerLemeshow (Hosmer \& Lemeshow 2000), donde valores inferiores a 0,05 indicaron mal ajuste del modelo. Los efectos de la multicolinealidad, evaluada a través de coeficientes factores de inflación de varianza $V I F>10$ (Hair et al. 1999), índice de condicionamiento $I C>25$, dimensión con carga superior a 0,50 (Belsley et al. 2004), realizando análisis de regresiones lineales siguiendo lógica popperiana (?). Según Garson (2015), se usaron medidas de bondad de ajuste (Hu \& 
Bentler 1999) $\chi^{2}$ (ausencia de significatividad refleja buen ajuste global de datos con las relaciones propuestas), SRMR (valores por debajo de 0,05), CFI (valores superiores a 0,95), TLI (superior a 0,95), RMSEA (inferior a 0,05). Se empleó procedimiento de estimación asintóticamente libre $(\mathrm{ADF})$, no requerida distribución normal multivariante.

En lo concerniente a actitudes hacia formas colaborativas de participación, se utilizó el indicador de Inglehart (1977) en su escala para medir valores, reducida a 3 ítems; se preguntó al entrevistado por primer y segundo objetivo en importancia que la institución debía plantearse en los siguientes diez años para mejorar el aprendizaje creativo en los programas académicos y en la calidad de estos, cuáles considera son las necesidades institucionales para mejorar la creatividad de los estudiantes? qué acciones propone para revitalizar la enseñanza en el aula? Preguntas que buscan vincular la juventud en la toma de decisiones a través de la lógica de la consulta (Parés 2009). La ponderación de cada dimensión e indicador, de acuerdo con el método análisis factorial, otorga inicialmente igual peso a cada una de las dimensiones.

Respecto a variables de tipo cualitativo, se busca a través del instrumento CREA una aproximación global a situaciones sociales con el fin de explorarlas, describirlas (Bourguignon et al. 2013) a partir de una postura epistemológica de tipo históricohermenéutica, método narrativo, por medio de la interpretación, que comienza como hipótesis para transformarse en tesis (Beuchot 2009). Mediante preguntas abiertas, los relatos, expresados en las narrativas, buscan identificar percepciones de los jóvenes sobre la categoría creatividad, en aras de enriquecerla y resignificar desde su lenguaje y forma de vida cotidianas, ampliando las concepciones. Se conversó con jóvenes individualmente para escuchar sus narrativas, a través de ítems propios de una entrevista semiestructurada, a fin de favorecer el carácter de las descripciones coloquiales, frente a las descripciones técnicas (White \& Epston 1993). Se realizó para esta dimensión una primera evaluación cualitativa del cuestionario en la que los participantes suprimieron e incorporaron ítems y aportaron sugerencias de redacción; en una segunda evaluación se reenvía el nuevo cuestionario para que ponderaran la importancia o valor de los ítems en una escala ordinal de 5 grados, calculándose la varianza de cada ítem y la global. Con el instrumento resultante se realizó un estudio de la fiabilidad para esta escala en el que se valoró la consistencia interna y la concordancia intraobservador. Para ello se seleccionaron entrevistas procedentes de la base de entrevistas videograbadas con objetivos de investigación. Un experto evaluó estas entrevistas en dos ocasiones con intervalo de evaluación de dos meses (procedimiento test-retest).

Se estructuró un modelo cuyos estadísticos considerados para valorar el ajuste del modelo estructural fueron el índice de bondad del ajuste realizado (AGFI), y el índice de bondad del ajuste (GFI), que miden el porcentaje de la matriz de varianzas y covarianzas explicado por las distintas ecuaciones del modelo estructural. El estadístico AGFI tiene en cuenta el número de grados de libertad con relación al número de indicadores que intervienen. También se muestran los valores de los estadísticos alternativos propuestos por Hu \& Bentler (1999) y Hair et al. (1999) 
normed fit index (NFI) y comparative fit index (CFI), así como del índice de ajuste incremental (IFI) de Bollen, el cual proporciona una medida de ajuste relativa al comparar las chi-cuadrado del modelo estimado y de un modelo de referencia en el que todas las variables observables están correlacionadas.

El análisis estadístico de validación determina parámetros de consistencia interna del test, índice kappa de Cohen ponderado (índice de concordancia simple), coeficiente de correlación intraclase (CCI) (según variables cualitativas o cuantitativas) para determinar la fiabilidad intraobservador utilizando SPSS 22 y AMOS. Además, se aplicó el test de Kolmogorov-Smirnov para comprobar si los valores de la escala se ajustaban a una distribución normal, prueba de la $t$ de Student para comparación de medias para datos emparejados, y método de Bland y Altman para analizar la concordancia intraobservador de forma descriptiva. Todos los contrastes de hipótesis fueron bilaterales, para $p<0,05$.

\section{Resultados}

Se obtuvo información completa sobre la evaluación del aprendizaje creativo de 595 jóvenes estudiantes de programas académicos de ingeniería, administración de empresas, ciencias agrarias. Entre estos grupos no hubo diferencias significativas según edad $(p=0,393)$, escolaridad $(p=0,796)$, con mayor predomino de mujeres entre casos $(p=0,017)$. Aplicaciones reales, satisfacción emocional, práctica pedagógica orientada al liderazgo, problemas propuestos, recursos en el aula son aspectos más mencionados cuando se pregunta por los cinco más influyentes en su satisfacción. Referente a indicadores de MIDE, estudiantes no se encuentran vinculados a proyectos de investigación, solo tres estudiantes pertenecen a semilleros de investigación, no registran productos, se cuenta con dos profesores con título de doctorado.

Los índices de bondad de ajuste muestran resultados chi-cuadrado $=45,19, D F=$ 0,43, p-valor < 0,02. TLI : 0,965; CFI : 0,971; SRMR =0,027; RMSEA : 0,$023 ; 1,6<V I F<2,8$; con $(R)=19$. Los resultados muestran que no existen problemas de multicolinealidad; controlando efectos bidireccionales o influencia de otras variables, la participación asociativa no se revela como predictor de variables dependientes; luego, participar en asociaciones no tiene efectos estadísticamente significativos en aprendizaje creativo, las medidas de ajustes del modelo reflejan resultados consistentes. En la aplicación de la prueba de Hosmer-Lemeshow pvalor $=0,887$, implica que lo observado se ajusta a lo esperado, hay alta proximidad entre valores reales y teóricos, luego usar el modelo y calcular predicciones con él es correcto, considerándose como una calibración.

El test de no aditividad de Tukey confirmó la aditividad de la escala $(p>0,05)$, que presentó consistencia interna de 0,89 en productividad, consistencia interna de 0,95 en independencia, 0,91 en motivación, 0,967 en flexibilidad, 0, 87 en actitud creativa; satisfacción con su integración social y satisfacción con sus recursos emocionales, en el contexto, mostraron un efecto relevante en el aprendizaje creativo. 
La consistencia de la medición muestra que la escala funciona de manera similar bajo diferentes condiciones, dependientes del mismo instrumento, del tiempo de aplicación, del que hace la medición, de los sujetos, de la interacción entre estas fuentes y del error aleatorio. La consistencia interna del instrumento muestra que los ítems miden el mismo concepto, en este caso, aprendizaje creativo; Alpha de Cronbach de 0,93 muestra que la escala es homogénea. De igual manera, al analizar alfa por elemento, los ítems dentro de la escala son complementarios el uno al otro, la escala es unidimensional, mide solo concepto. Asimismo, Alpha basada en elementos estandarizados 0,91 muestra alta homogeneidad y fiabilidad del instrumento. El nivel de covariación de los ítems entre si y la cantidad de ítems o partes que conforman la prueba.

Análisis de varianza muestra una media de 7 y varianza 3,2 para 595 elementos, Fisher de 2,6, p-valor $=0$ entre elementos, estimación de Tukey de potencia en la que se deben realizar observaciones para conseguir una aditividad de 3, 925 . Se puede decir que la confiabilidad mide el error que genera un instrumento al ser inestable y aplicarse en diferentes ocasiones. El coeficiente de correlación 0, 89 muestra estabilidad temporal y consistencia en respuestas de los individuos. Para la estabilidad del instrumento, se observa un coeficiente de reproductibilidad $C R=0.899$; escala de Gutman cercana alpha de cronbach mostrando fiabilidad y estabilidad del instrumento $(\lambda 0,937,0,890 ; 0,879 ; 0,861 ; 0,898, \cdots$ para 35 elementos).

Validez convergente por factores latentes productividad $(\alpha=0,76$, validez $=0,72)$, independencia $(\alpha=0,9$; validez $=0,86)$, motivación $(\alpha=0,86$; validez $=0,82)$, actitud creativa $(\alpha=0,86$; validez $=0,76)$, satisfacción emocional $(\alpha=0,72$; validez $=0,55)$, pensamiento creativo $(\alpha=0,86$; validez $=0,75)$, competencias de liderazgo $(\alpha=0,9$; validez $=0,86)$, práctica orientada al liderazgo $(\alpha=0,77$; validez $=0,7)$.

La reproductibilidad marginal mínima 0, 912, efecto de reactivos que se distribuyen con valor modal extremo. El coeficiente de escalabilidad de 0,153 indica escala no acumulativa, mostrando reproductibilidad del instrumento para toma de decisiones en conjunto de todos los ítems por factor. Lo anterior muestra que el instrumento es estable, es decir, se obtienen los mismos resultados al utilizar el instrumento en condiciones similares en diferentes ocasiones y es factible generalizar resultados. En modelo de efectos combinados bidireccionales donde los efectos de personas son aleatorios y los efectos de medidas son fijos, el coeficiente de correlación se muestra superior a 0,67, Fisher $=90,097$, p-valor igual a 0, mostrando fiabilidad aceptable para medidas únicas y alta para medidas promedio. Para equivalencia, prueba de bondad de ajuste modelo de formas paralelas, chi-cuadrado de $-45,6$, varianza 0,5 ; correlación 0,7523; fiabilidad escala 0,898; fiabilidad sin sesgo 0,98 Coeficientes de correlaciones entre clases tipo $\mathrm{C}$ utilizando una definición de coherencia.

Análisis de validez de constructo determina cada coeficiente de acuerdo entre evaluadores con nivel según los evaluadores 1 y 2,1 y 4, Kappa $=0,9687$, error 0, 03 $T=14.5 p=0$; nivel de acuerdo evaluadores 1 y 5,2 y 3,3 y 4,4 y 5,4 y 7 Kappa $=0,898$, error $0,03, T=12,8 p=0$; nivel de acuerdo evaluadores 1 y 3 , 
3 y 5 , 1 у 6, 1 y 7 Kappa=0,868, error $0,03 \mathrm{p}=0$; nivel de acuerdo evaluadores 5 y 2,5 у 6,2 у 4, Kappa $=0,837$, error $0,04 \mathrm{p}=0$; nivel de acuerdo evaluadores 6 у 4, 6 у 7, 2 у 6, 2 у 7 Kappa=0,98, error $0,04 \mathrm{p}=0$; nivel de acuerdo evaluadores 5 y 7, 6 у 3 Kappa=0,968, error 0,01 p=0; nivel de acuerdo evaluadores, 3 у 7 $\mathrm{Kappa}=0,98$, error $0,02, \mathrm{p}=0$. El valor de Kappa es estadísticamente significativo diferente de cero (Cohen 1960) y teniendo en cuenta valoración según Landis \& Koch (1977) su valor Kappa (0,91) sugiere que las calificaciones de los expertos son muy similares, existiendo un acuerdo fuerte casi perfecto entre ellos, es decir, una fuerza de concordancia muy buena. Se concluye que la escala es válida. El análisis factorial arrojó siete dimensiones, consistencia interna adecuada (alfa de cronbach 0,81-0,9), promedio de las diferencias en la sumatoria de ítems -1,6 (DS: 8,56, límites de acuerdo de Bland y Altman fueron -16,9 y 12,3.

Se encontraron correlaciones significativas entre las distintas pruebas que conforman el instrumento, lo que sugería el uso del análisis factorial exploratorio confirmado también por la prueba de esfericidad de Bartlett $\left(\chi^{2}=1717,9, g l=34\right.$, $p=0)$, índice Kayser-Meyer-Olkin $(\mathrm{KMO}=0,879)$ para detección de dimensiones subyacentes, y comparar la estructura factorial encontrada con estudio de Chaparro et al. (2006).

En aplicación de subescalas se asocian categorías en un modelo jerárquico $8 \times 2$, con indicadores de productividad, independencia, motivación, actitud creativa, satisfacción emocional, con subescala: rol, autoestima, reconocimiento, autonomía y afrontamiento; competencias de liderazgo, con subescala: liderazgo en relaciones, tareas, cambio, capacidades; pensamiento creativo con subescala: fluidez, originalidad, flexibilidad, flexibilidad graficar-contenido verbal, contenido figurativo; práctica pedagógica con subescala trabajo grupal, escrito, presentaciones, prácticas, construcción de prototipos, generación de modelos, uso de tecnologías de la información, generación de material como videos, blogs) y la covariancia de los errores con el mismo contenido.

El modelo estructural (figura 1) presentó niveles de ajuste apropiados (AGFI= 0,939, $\mathrm{GFI}=0,95$, Bollen's $\mathrm{IFI}=0,945, \mathrm{Qui}=971, \mathrm{df}=62, \mathrm{p} ; 0,001, \mathrm{CFI}=0,971$, $\mathrm{NFI}=0,973$, RMSEA = 0,038), asumiéndose como una forma de analizar la estructura subyacente al desempeño de los estudiantes frente a su aprendizaje creativo en sub-tests. Efectos totales estandarizados de la participación asociativa sobre coeficiente Bca 95\% CI inferior superior satisfacción con institución y enfoque pedagógico 0,04, satisfacción con actividades que fomenten creatividad 0,21, satisfacción con su integración social 0,16, satisfacción con sus recursos emocionales 0,11; V de Cramer 0,16. La raíz cuadrada del error cuadrático medio (RMSEA), evidencia que tanto la estimación puntual como el intervalo de confianza confirman un elevado grado de ajuste del modelo estimado a los datos.

Los resultados sugirieron una estructura estable de ocho factores para el aprendizaje creativo $A_{i j}$; tres factores de primer orden correlacionados: pensamiento creativo $(P c)$ factor principal, práctica pedagógica orientada al liderazgo $(\rho)$, satisfacción emocional $(S c)$; factores de segundo orden: actitud creativa $(a c)$, productividad $(p)$, independencia $(i)$, competencias de liderazgo $(c l)$, motivación $(M)$ y 


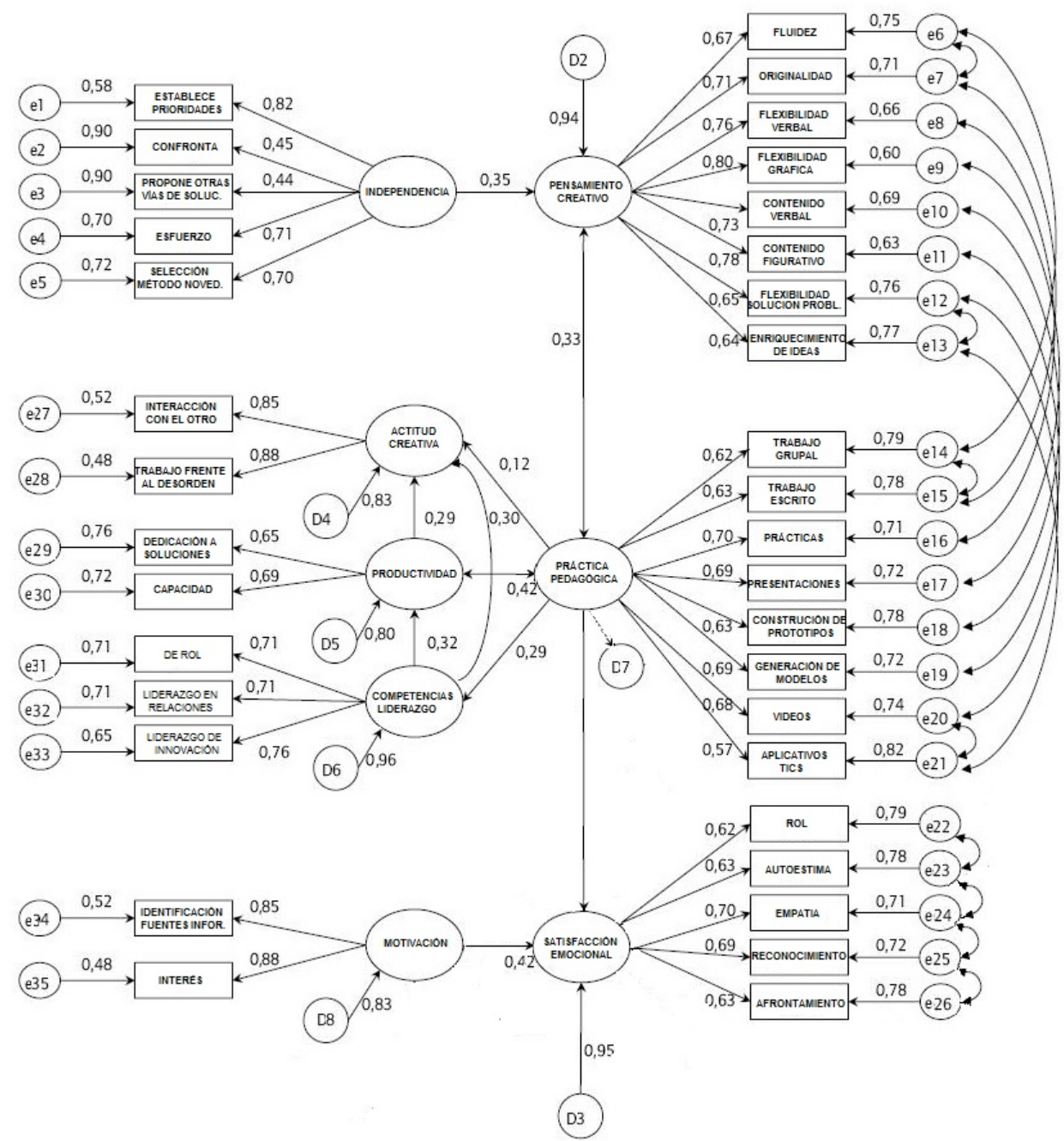

Figura 1: Modelo estimado. AMOS. Fuente: elaboración propia.

35 variables $X$ : fluidez, originalidad, flexibilidad verbal, flexibilidad gráfica, contenido verbal, contenido figurativo, solución de problemas, enriquecimiento de ideas; trabajo grupal, trabajo escrito, prácticas, presentaciones, construcción de prototipos, generación de modelos, videos, uso aplicativos; rol, autoestima, empatía, reconocimiento, afrontamiento; prioridades, confrontación, proposición soluciones, esfuerzo, selección de métodos novedosos; interacción con el otro, trabajo frente al desorden, dedicación a soluciones, capacidad, de rol, liderazgo de relaciones, liderazgo de innovación, identificación fuentes de información, interés hacia la creación. 
Las ecuaciones encontradas son:

$$
A_{i j}=0,9 c_{j}+0,95 S e_{j}+0,33 \rho_{j}+0,038
$$

donde

$$
\begin{aligned}
P c_{j} & =0,35 i_{j}+X_{2 j} \\
S e_{j} & =M_{j}+X_{3 j} \\
\rho_{j} & =0,12 a c_{j}+0,29 c l_{j}+0,42 p_{j}+X_{7 j}+0,3+\sum_{4}^{6} X_{7 j}
\end{aligned}
$$

En torno a la validez facial, la idoneidad del contenido, según Secolsky (1987), muestra un instrumento que cumple con parámetros de claridad $(I C=0,85)$, precisión $(I C=0,935)$ y comprensión $(I C=0,937)$. De esta manera cada ítem cumple con el criterio de la categoría, está redactado de forma exacta, de fácil comprensión, permite interpretar y entender su significado

\section{Agradecimientos}

La investigación fue realizada parcialmente con apoyo financiero otorgado por el Fondo de Investigación Universitario FINU de la Universidad Francisco de Paula Santander, a través de proyecto de investigación "Impacto de la comunidad de aprendizaje, en el desarrollo de competencias e indicadores de innovación en la enseñanza del cálculo diferencial", contrato de cofinanciación 021-2016 y tesis de maestría en prácticas pedagógicas: "Estrategia pedagógica en física mecánica basada en el aprendizaje creativo".

Recibido: 28 de marzo del 2016

Aceptado: 23 de abril del 2016

\section{Referencias}

Ang, S. \& Low, S. (2000), 'Exploring the Dimensions of ad Creativity', Psychology and Marketing 17(10), 835-854.

Belsley, D., Kuh, E. \& Welsch, R. (2004), Regression diagnostic: Identifiying influential data and sources of collinearity, Hoboken, New Jersey: John Wiley.

Bermejo, R., Hernández, D., Ferrando, M., Soto, G., Sainz, M. \& Prieto, M. (2010), 'Creatividad, inteligencia sintética y alta habilidad', Reifop 13(1).

Beuchot, M. (2009), Tratado de hermenéutica analógica. Hacia un nuevo modelo de interpretación., México: UNAM-Ítaca. 
Bourguignon, F., Ferreira, F. \& Menéndez, M. (2013), Inequality of Opportunity in Brazil: A Corrigendum, Economics Papers from University Paris Dauphine 123456789/11396. Paris: Dauphine University. .

Brown, J., Bowling, A. \& Flynn, T., eds (2004), Models of quality of life: A taxonomy, overview and systematic review of the literature., European Forum on Population Ageing Research., The address of the publisher.

*http://www.shef.ac.uk/ageingresearch/pdf/qol eview $_{c}$ omplete.pdf

Cabrera, J. \& de la Herrán, A. (2015), 'Creatividad, complejidad y formación: un enfoque transdisciplinar', Revista Complutense de Educación 26(3), 505-526.

Campbell, A., Converse, P. \& Rogers, W. (1976), The quality of American life: perceptions, evaluations and satisfaction, New York: Russell Sage.

Chaparro, F., Ortiz, F. \& Bruges, M. (2006), Formulación de una estrategia de ciudad región del conocimiento para Bogotá. Una propuesta de acción., Bogotá: Universidad del Rosario. Departamento Administrativo de Planeación Distrital (DAPD).

Cramond, B., Matthews-Morgan, J., Bandalos, D. \& Zuo, L. (2005), 'Report on the 40-year follow up of the torrance tests of creative thinking: Alive and well in the new millennium', Gifted Child Quarterly 49(4), 283-293.

Csikszentmihalyi, M. (1996), Creativity: Flow and the Psychology of discovery and invention, New York: Harper Perennial.

Dutta, S., Lanvin, B. \& Wunsch-Vincent, S. (2015), The Global Innovation Index 2015. Effective Innovation Policies for Development, Cornell University, INSEAD, and WIPO.

Florida, R., Balcells, R. \& Arnau, A. (2009), Les Ciutats creatives: com L'economia està convertint la tria de l'indret on viure en la decisió més important de la teva vida., Barcelona: Pòrtic.

Garaigordobil, M. \& Pérez, J. (2002), 'Análisis predictivo y correlacional de la creatividad gráfica y verbal con otros rasgos de la personalidad infantil', Revista de Psicología General y Aplicada 55(3), 373-390.

Gardner, H. (1983), Frames of mind., New York: Basic Books.

Gardner, H. (1999), Mentes Creativas, Barcelona: Paidós.

Garson, G. (2015), Structural Equation Modeling., Asheboro, NC: Statistical Associates Publishers.

González, C. (2008), El proceso creativo en el diseño arquitectónico. Encuentros Multidisciplinares, Fundación General de la Universidad Autónoma de Madrid. Madrid, España.

Green, A. (2010), Creativity in public relations., London: Crest Publishing House. 
Hair, J., Anderson, R., Tatham, R. \& Black, W. (1999), Análisis Multivariante, Madrid: Prentice Hall.

Hosmer, D. \& Lemeshow, S. (2000), 'A goodness-of-fit test for the multiple logistic regression model', Communications in Statistics 10(1), 1043-1069.

Hu, L. \& Bentler, P. (1999), 'Cutoff criteria for fit indexes in covariance structure analysis: Conventional criteria versus new alternatives.', Structural Equation Modeling 6(1), 1-55.

Inglehart, R. (1977), The Silent Revolution, Changing values and Political Siyles among Western Publics, Princeton: Princeton University Press.

Kumar, V., Kemmler, D. \& Holman, E. (1997), ' The creativity styles questionnaire?Revised.', Creativity Research Journal 10(1), 51-58.

Landry, C. \& Hyams, J. (2012), The creative city index: Measuring the pulse of the city, New Stroud: Gloucestershire/GB.

Martínez, J., Vergel, M. \& Zafra, S. (2015), Comportamiento juvenil y competencias prosociales, Bogotá: Ibáñez.

Morales, P., Urosa, B. \& Blanco, A. (2003), Construcción de escalas de actitudes tipo Likert., Madrid: La Muralla.

Oyekale, T., Okunmadewa, F., Omonona, B. \& Oni, O. (2009), 'Fuzzy Set Approach to Multidimensional Poverty Decomposition in Rural Nigeria', The IUP Journal of Agricultural Economics 6(3), 7-44.

Pardo, A. \& Ruiz, M. A. (2002), SPSS 11. Guía para el análisis de datos, Madrid: McGraw-Hill.

Parody, G. (2015), El ranking de las universidades: Miremos más allá del ombligo., El Tiempo, 23 de julio.

Parés, M. (2009), Introducción: participación y evaluación de la participación. En M. Parés (coord.). Participación y calidad democrática. Evaluando las nuevas formas de democracia participativa., Barcelona: Ariel.

Prieto, M., Fernández, G., Rojo, F., Lardiés, R., Rodríguez, V., Ahmed, K. \& Rojo, J. (2008), 'Factores sociodemográficos y de salud en el bienestar emocional como dominio de calidad de vida de las personas mayores en la comunidad de madrid, 2005.', Revista Española de Salud Pública 82(3), 301-313.

Secolsky, C. (1987), 'On the direct measurement of face validity: A comment on nevo.', Journal of Educational Measurement 24(1), 82-83.

Sternberg, R. (2006), 'The Nature of Creativity', Creativity Research Journal 18(1), 8798.

Sáenz, D. \& Larraz, N. (2015), 'El pensamiento creativo en la escuela'. 
Torrance, E. (1976), Tests de Pensée Créative de E.P. Torrance: Manuel., París: Centre de Psychologie Appliquee.

Vergel, M., Martínez, J. \& Zafra, S. (2015), 'Validez de instrumento para medir la calidad de vida en la juventud: VIHDA', Revista Logos Ciencia \& Tecnología 7(1), 2028.

Vivant, E. (2013), 'Creatives in the city: Urban contradictionsof the creative city', City, Culture and Society 4(2), 57-63.

Wallack, M. A. \& Koga, N. (1965), Modes of thinking in children, New York: Holt, Rinehart y Winston.

Wasserman, M. (2015), Calidad de la educación. ¿Qué mide el Mide?, El Tiempo, 24 de julio.

White, M. \& Epston, D. (1993), Medios narrativos para fines terapéuticos., Barcelona: Paidós. 